\title{
Experimental Design of Exit Wave Reconstruction from a Transmission Electron Microscope Defocus Series
}

\author{
Martijn A. O. Miedema, Adriaan van den Bos, and A. H. Buist
}

\begin{abstract}
Recently published methods reconstruct the complex exit wave of the specimen in a transmission electron microscope by combining a number of images recorded at different defocus values. An expression is derived for the variance of the reconstructed wave as a function of the experimental parameters that can be freely chosen. It is shown how these parameters can be used for experimental design, that is, for minimizing the variance of the reconstructed wave.
\end{abstract}

\section{INTRODUCTION}

A TRANSMISSION electron microscope (TEM) is an important instrument for investigating specimens up to the atomic level. Problems connected with linking the images to the specimen structure are the linear distortion of the electron exit wave of the specimen by the microscope and the loss of the phase angle information in the recording process. Several authors have proposed a method of exit wave reconstruction by means of a defocus series to overcome the above problems [4], [9], [13]. Practical results have already been demonstrated in [1], [2], [5], [9].

The nonlinear imaging model for a TEM may be linearized for a wide range of specimens. This linearization is also used in this paper. Because the pixel intensities of the recorded image are Poisson variates, the reconstruction problem has to be viewed as a statistical estimation problem. It is investigated how the free parameters in the design of the experiments [3] should be chosen in order to optimize the estimates of the exit wave under the constraint of a limited electron dose.

\section{IMAGE RECONSTRUCTION}

Image formation for a TEM is described in terms of the complex electron exit wave $\varphi_{\mathrm{ex}}(\vec{r})$, which contains the information about the specimen, and the complex, defocus dependent microscope impulse response function $h(\vec{r}, z)$. The $\vec{r}=(x, y)$ plane is perpendicular to the optical axis $\mathrm{z}$ of the microscope. In quasi-coherent and linear approximation, the image intensity is described by [8]:

$$
I(\vec{r}, z)=I_{0}(z)\left[1+\varphi_{\mathrm{ex}}(\vec{r}) * h(\vec{r}, z)+\varphi_{\mathrm{ex}}^{\prime}(\vec{r}) * h^{\prime}(\vec{r}, z)\right]
$$

Manuscript received May 18, 1993; revised November 17, 1993. This paper was presented at the Instrumentation and Measurement Technology Conference, Irvine, CA, May 18-20, 1993.

The authors are with the Department of Applied Physics, Delft University of Technology, 2600 GA Delft, The Netherlands.

IEEE Log Number 9215985 with' the complex conjugate, * a two-dimensional convolution, $I_{0}(z)$ the average electron dose, and $\varphi_{\mathrm{ex}}(\vec{r})$ is zero mean. Solving (1) for the exit wave means deconvolving with respect to the impulse response function, which is easier to perform in the frequency domain. Splitting up the exit wave in its real $\varphi_{\mathrm{re}}(\vec{r})$ and imaginary part $\varphi_{\mathrm{im}}(\vec{r})$, and taking the Fourier transform of (1), yield the linear expression for the image spectrum [7]:

$$
\begin{gathered}
I(\vec{g}, z)=I_{0}(z)\left[\delta(\vec{g})+2 \Phi_{\mathrm{re}}(\vec{g}) \operatorname{Re}[H(\vec{g}, z)]\right. \\
\left.\cdot 2 \Phi_{\mathrm{im}}(\vec{g}) \operatorname{Im}[H(\vec{g}, z)]\right] .
\end{gathered}
$$

Here $\vec{g}=\left(g_{x}, g_{y}\right)$ is the two-dimensional spatial frequency, and $\delta(\vec{g})$ and is a Dirac pulse. The spectra $\Phi_{\mathrm{re}}(\vec{g})$ and $\Phi_{\mathrm{im}}(\vec{g})$ are the Fourier transform of the real and imaginary part of $\varphi_{\mathrm{ex}}(\vec{r})$, respectively. From now on, the term exit wave spectrum refers to this pair of spectra. They are usually associated with the spectrum of an amplitude and phase specimen, respectively. The microscope transfer function $H(\vec{g}, z)$ is defined by [14]:

$$
H(\vec{g}, z)=E_{s p}(\vec{g}, z) E_{c h r}(\vec{g}) \exp (j \chi(\vec{g}, z))
$$

with $E_{s p}(\vec{g}, z)$ the real-valued spatial incoherency envelope function, $E_{c h r}(\vec{g})$ the real-valued temporal incoherency envelope function, and $\chi(\vec{g}, z)$ the real-valued wave aberration function. Direct reconstruction from one image of an amplitude or phase specimen is impossible because zeros in the respective transfer functions render the problem ill-conditioned. A defocus series is a set of $N$ images at different, known defocus positions along the optical axis. From this set of real-valued images the complex-valued exit wave of the specimen can be reconstructed. This requires at least two images. Because the positions of the zeros of the transfer function of the microscope change with defocus, the defoci can be chosen such that frequencies absent in the Fourier spectrum of the one image are present in that of the other. Thus ill-conditioning of the reconstruction process is avoided.

Per spatial frequency $\vec{g}$, a set of $N$ linear equations, $I\left(\vec{g}, z_{n}\right), n=1, \cdots, N$, is obtained where each equation corresponds to a different defocus position. Because $\Phi_{\mathrm{re}}(0)=$ $\Phi_{\text {im }}(0)=0$ (zero mean values), the terms for $\vec{g}=0$ may be omitted and in matrix notation the set of equations is written as $J=G \Phi$. Here $J$ is the $N \times 1$ vector with the image (intensity) spectrum at $N$ different defoci, $G$ is the $N \times 2$ matrix with the real and imaginary parts of the transfer function at $N$ different 
defoci, and $\Phi$ is the $2 \times 1$ vector with the exit wave spectrum. Solving this overdetermined set of equations for the exit wave spectrum gives the general solution

$$
\Phi=\left(G^{H} G\right)^{-1} G^{H} J
$$

with $H$ the complex conjugate transpose. A solution exists only if the determinant of $G^{T} G$ is nonzero for all $\vec{g}$. If the defocus steps $\Delta z(n)$ between the images are equal, it can be shown [7] that this condition is fulfilled when $\Delta z(n)<$ $1 /\left(\lambda g_{\max }^{2}\right)$, with $g_{\max }$ the highest spatial frequency present in the images and $\lambda$ the wavelength of the electrons. This gives a direct upper limit for the defocus step size between the images.

\section{ANALYSIS OF ERROR SOURCES}

Inherent to the process of counting electrons, the recording of TEM images introduces Poisson noise. In a modern Charged Coupled Device (CCD) based camera, the Poisson noise is the dominant nonsystematical error source [1]. Other, systematical error sources, are: nonlinear imaging terms [5], inelastic scattering [10] and inaccuracies in measured defocus position and spherical aberration. Neglecting the latter, it is the Poisson noise that corrupts the measurements. When the number of electrons counted is large compared with the square root of this number, the Poisson distribution may be replaced by a normal one [6]. Then the measured intensities at pixel position $(k, l)$ are described by

$$
i\left(k, l, z_{n}\right)=I\left(k, l, z_{n}\right)+\epsilon\left(k, l, z_{n}\right)
$$

with expectation $\mu_{i}\left(k, l, z_{n}\right)=I\left(k, l, z_{n}\right)$. The $\epsilon\left(k, l, z_{n}\right)$ are uncorrelated, zero mean, normally distributed errors with variance $\sigma_{i}^{2}\left(k, l, z_{n}\right)=I\left(k, l, z_{n}\right)$. If the contrast in the images is low, which applies to a wide range of specimens, the variance at all pixels and per image may be approximated by the averaged intensity over all pixels, $\sigma_{i}^{2}\left(z_{n}\right)=I_{0}\left(z_{n}\right)$.

\section{PRECISION OF THE RECONSTRUCTION}

Because the recorded images are not free of noise, only an estimate of the exit wave spectrum is obtained, and the estimator determines the precision (standard deviation) of the estimates. Reconstruction is performed by a two-step estimation procedure:

1) Estimate the image spectrum at each defocus with a two-dimensional Discrete Fourier Transform (DFT).

2) Estimate the exit wave spectrum from the image spectra at different defoci.

Consider the first step. Suppose that the specimen has a periodical structure and that the processed images consist of an integer number of periods. Then the spectrum is the complex Fourier coefficient spectrum of the periodical image, and the DFT is equivalent to the ordinary least squares (OLS) estimator [12]. As stated before, the errors in each pixel are uncorrelated and normally distributed with the same variance. Then the OLS and, therefore, the DFT estimator have minimum variance among all estimators [11]. Also the points of the estimated spectrum are unbiased, noncovariant, normally distributed and have a common variance $I_{0}\left(z_{n}\right) / L^{2}$, with $L$ the number of pixels in the $x$ and $y$ direction [7]. In the second step, the exit wave spectrum is estimated from the estimated image spectra. Now the set of equations to be solved includes the errors $\epsilon\left(\vec{g}, z_{n}\right)$ :

$$
i\left(\vec{g}, z_{n}\right)=I\left(\vec{g}, z_{n}\right)+\epsilon\left(\vec{g}, z_{n}\right) \quad n=1, \cdots, N .
$$

Since the $\epsilon\left(\vec{g}, z_{n}\right)$ are normally distributed and uncorrelated, but have a different variance for different $n$, the most precise estimator for $\Phi_{\mathrm{re}}(\vec{g})$ and $\Phi_{\mathrm{im}}(\vec{g})$ is the weighted least squares estimator with the reciprocals of the variances of the $\epsilon\left(\vec{g}, z_{n}\right)$ as weights [7]. The solution to (6) for the exit wave spectrum for the frequency $\vec{g}$ is

$$
\hat{\Phi}=\left(G^{H} V^{-1} G\right)^{-1} G^{H} V^{-1} \hat{J} .
$$

Here $\hat{\Phi}$ is the $2 \times 1$ vector of the estimated exit wave spectrum, $\hat{J}$ the $N \times 1$ vector of the estimated image spectra at the $N$ different defoci, and $V$ the $N \times N$ diagonal covariance matrix with the variances $I_{0}\left(z_{n}\right) / L^{2}$ of the $\epsilon\left(\vec{g}, z_{n}\right)$ as its diagonal elements. The $2 \times 2$ covariance matrix of the estimated exit wave spectrum is equal to $\left(G^{H} V^{-1} G\right)^{-1}$. The diagonal elements of this matrix, which are the variances $\sigma_{\mathrm{re}}^{2}$ and $\sigma_{\mathrm{im}}^{2}$ of $\Phi_{\mathrm{re}}(\vec{g})$ and $\Phi_{\mathrm{im}}(\vec{g})$, respectively, are

$$
\begin{aligned}
\sigma_{\mathrm{re}}^{-2} & =4 L^{2} E_{c h r}^{2}(\vec{g}) \\
\times & \frac{\sum_{n=1}^{N-1} \sum_{m=n+1}^{N} f\left(z_{n}, z_{m}, \vec{g}\right) \sin ^{2}\left(\chi\left(\vec{g}, z_{n}\right)-\chi\left(\vec{g}, z_{m}\right)\right)}{\sum_{n=1}^{N} I_{0}\left(z_{n}\right) E_{s p}^{2}\left(\vec{g}, z_{n}\right) \sin ^{2}\left(\chi\left(\vec{g}, z_{n}\right)\right)}
\end{aligned}
$$

and

$$
\begin{aligned}
& \sigma_{\mathrm{im}}^{-2}=4 L^{2} E_{c h r}^{2}(\vec{g}) \\
& \times \frac{\sum_{n=1}^{N-1} \sum_{m=n+1}^{N} f\left(z_{n}, z_{m}, \vec{g}\right) \sin ^{2}\left(\chi\left(\vec{g}, z_{n}\right)-\chi\left(\vec{g}, z_{m}\right)\right)}{\sum_{n=1}^{N} I_{0}\left(z_{n}\right) E_{s p}^{2}\left(\vec{g}, z_{n}\right) \cos ^{2}\left(\chi\left(\vec{g}, z_{n}\right)\right)}
\end{aligned}
$$

where

$$
f\left(z_{n}, z_{m}, \vec{g}\right)=I_{0}\left(z_{n}\right) I_{0}\left(z_{m} E_{s p}^{2}\left(\vec{g}, z_{n}\right) E_{s p}^{2}\left(\vec{g}, z_{m}\right) .\right.
$$

These expressions show how the variances can be manipulated by selecting the free parameters. These are the number of images $N$, the defocus values $z_{n}$, and the distribution of the allowable dose of electrons (sum of the average intensities $\left.I_{0}\left(z_{n}\right)\right)$ over the images.

\section{DESIGN RECONSTRUCTION EXPERIMENTS}

In the design of the experiments a distinction is made between instrumental parameters and free parameters. The instrumental parameters were set as follows. The gun was a field emission gun (FEG). The acceleration voltage was $300 \mathrm{kV}$. The spherical aberration was $1 \mathrm{~mm}$. The objective 


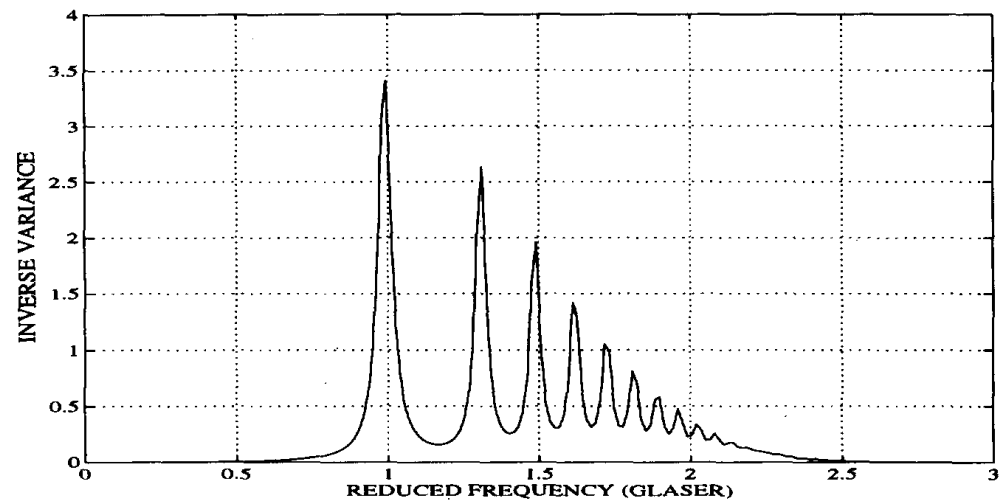

Fig. 1. Inverse normalized variance of the spectrum of the imaginary part of the exit wave for 2 images, start defocus -0.05 Sch and defocus step 0.1 Sch.

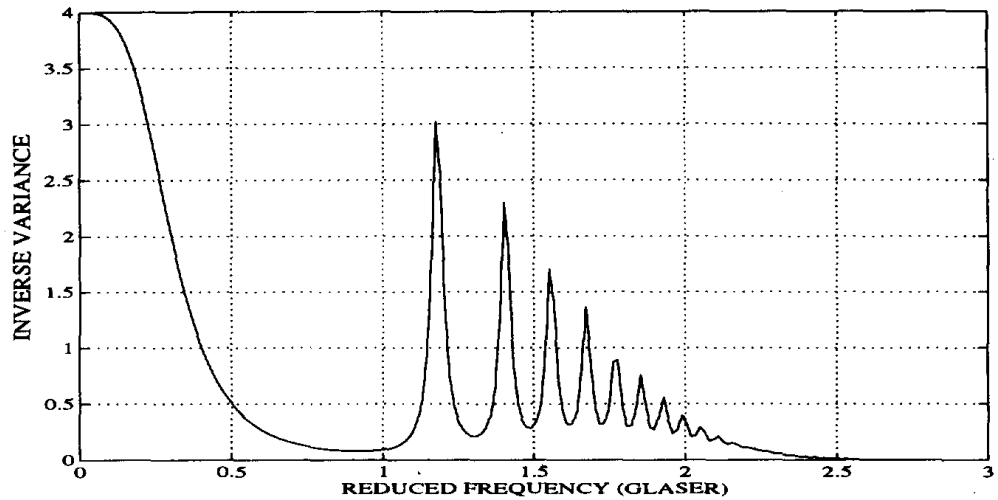

Fig. 2. Inverse normalized variance of the spectrum of the real part of the exit wave for 2 images, start defocus -0.05 Sch and defocus step 0.1 Sch.

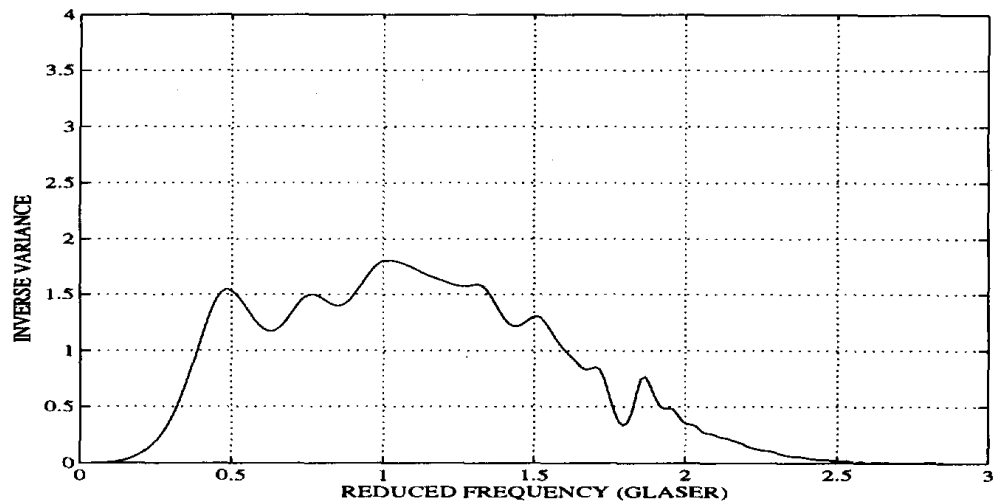

Fig. 3. Inverse normalized variance of the spectrum of the imaginary part of the exit wave for 16 images, start defocus 0 Sch and different defocus steps.

aperture was $20 \mathrm{mrad}$. The illumination angle was $0.1 \mathrm{mrad}$. The chromatic defocus spread was $100 \AA$ and there was no astigmatism. This gives a spatial resolution of about $1 \AA$. Also, $H\left(\vec{g}, z_{n}\right)[8]$, and therefore $\sigma_{\mathrm{re}}^{2}$ and $\sigma_{\mathrm{im}}^{2}[7]$, are rotationally symmetric with $\vec{g}$, and the figures shown below are given one-dimensionally as a function of $|\vec{g}|$.

Once the instrumental parameters are set, the free parameters as mentioned in Section IV are left. First, consider the electron dose; it is kept constant and is evenly distributed over all images. Uneven distribution has been shown to give suboptimal results and will therefore not be considered henceforth. In the first design, $N=2$, the start defocus is 0.05 scherzer (Sch) [8] (where 1 Sch corresponds to $44.2 \mathrm{~nm}$ for the above instrumental parameters), and the step size is equal to the maximum allowable value (as in Section II). For a resolution up to $1 \AA$ and the above TEM parame- 


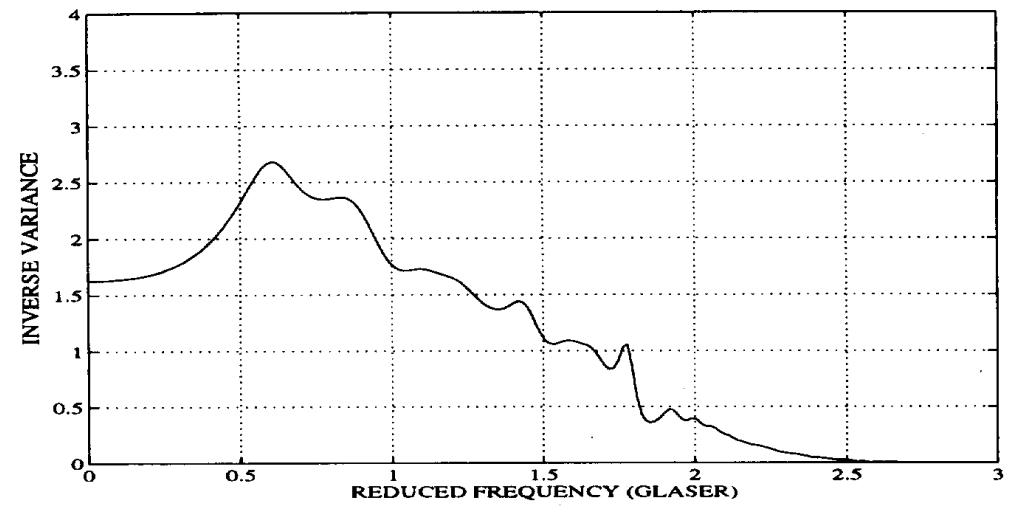

Fig. 4. Inverse normalized variance of the spectrum of the real part of the exit wave for 16 images, start defocus 0 Sch and different defocus steps.

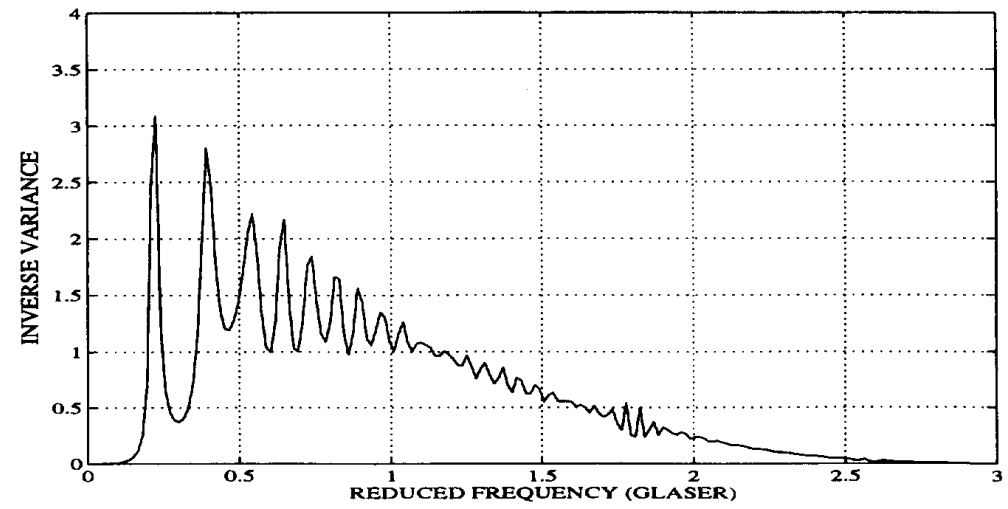

Fig. 5. Inverse normalized variance of the spectrum of the imaginary part of the exit wave for 16 images, start defocus 8 Sch and different defocus steps.

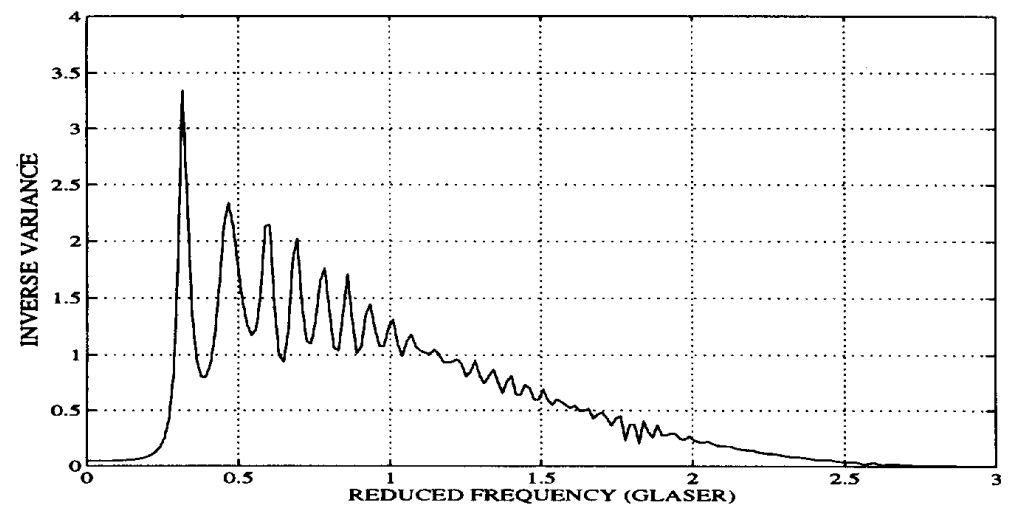

Fig. 6. Inverse normalized variance of the spectrum of the real part of the exit wave for 16 images, start defocus 8 Sch and different defocus steps.

ters, the maximum defocus step may be shown to be 0.1 Sch [7].

In the Figs. 1 and 2, the inverses of the variances of the estimated spectra of the real and imaginary part of the exit wave are plotted. The variance is normalized with respect to the total electron dose and the number of pixels, and the frequency coordinates are measured in glaser (G1) [8] (3 Gl corresponds to the above TEM parameters for a spatial resolution of $0.98 \AA$ ). Next, the number of images is increased to $N=16$, while the total dose is kept the same. Also the defocus steps are chosen differently, 0.1 Sch between the first 8 images and $0.3 \mathrm{Sch}$ between the last 8 images. The mix 


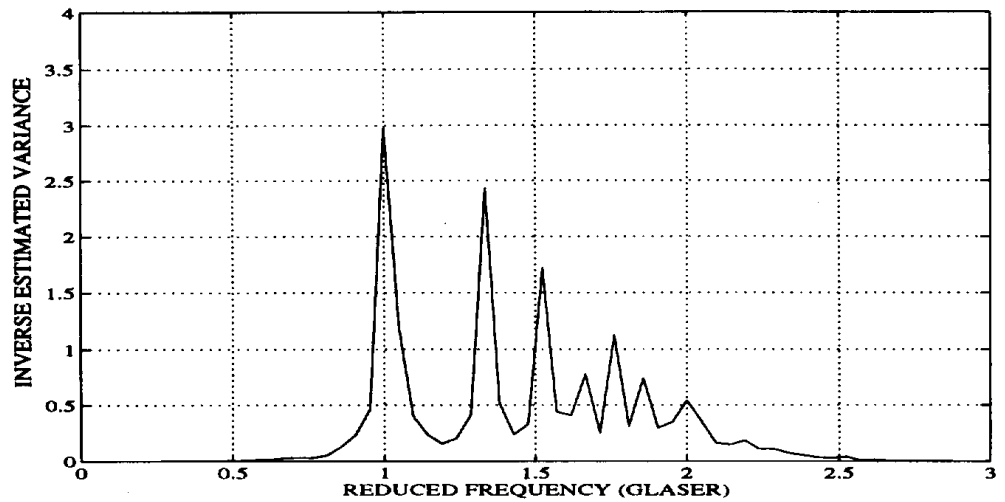

Fig. 7. Inverse of the estimated normalized variance of the spectrum of the imaginary part of the exit wave for 2 images, start defocus -0.05 Sch and defocus step 0.1 Sch.

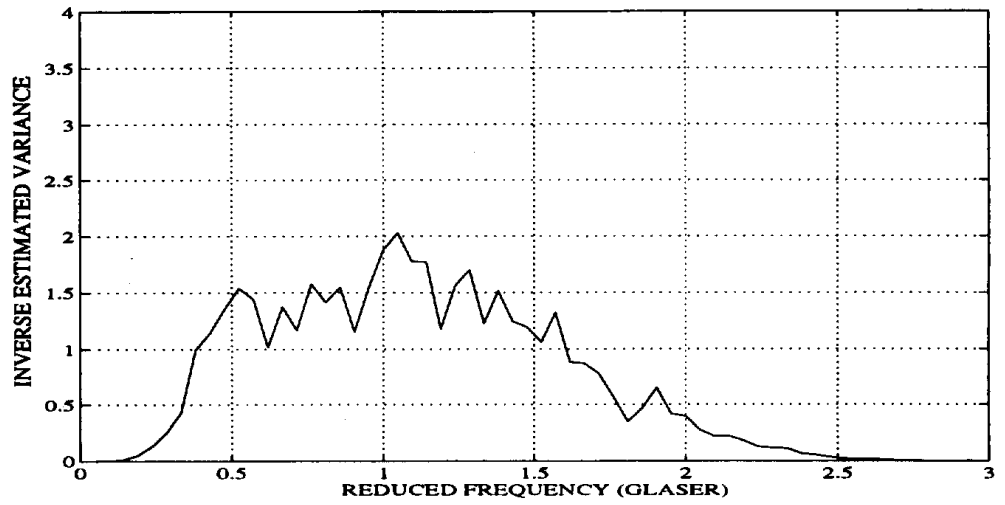

Fig. 8. Inverse of the estimated normalized variance of the spectrum of the real part of the exit wave for 16 images, start defocus 0 Sch and different defocus steps.

of different values prevents the ill-conditioning of the system described in Section II. Figs. 3 and 4 show the resulting inverse variances.

Clearly, it is advantageous to distribute the total electron dose over a relatively large number of images. The spectra can be accurately estimated over a broader band of frequencies, despite the fact that the SNR per image is much lower. Also it is seen that the estimate of the spectrum of the imaginary part of the exit wave gives very high variances for low spatial frequencies, which is due to the nature of image formation for phase objects [8]. Lastly, the choice of start defocus is investigated. Start defocus is $8 \mathrm{Sch}, N=16$, the first 8 defocus steps are $0.1 \mathrm{Sch}$, and the last 8 are $0.3 \mathrm{Sch}$. Figs. 5 and 6 show the results, illustrating the tradeoff in precision between the real and imaginary part much more clearly. Also, there is a strong, unwanted, oscillation in the precision as a function of $|\vec{g}|$.

From the above calculations it is concluded that the choice of the free parameters greatly influences the precision of the estimates. The choice should be based on the type of specimen and the required passband characteristics.

\section{SIMULATION EXPERIMENTS}

A number of simulation experiments have been done to verify the theoretical results above. In each of the experiments, which were all repeated 100 times, an image, based on the linear imaging theory (1), of a flat power carbon film was simulated for different defoci, to which white noise was added. Fig. 7 shows the inverse of the estimated normalized variance of the spectrum of the imaginary part of the exit wave for reconstruction from $N=2$, start defocus -0.05 Sch and defocus step $0.1 \mathrm{Sch}$. It corresponds to Fig. 1 and shows the agreement with the theoretically calculated variance.

Next, the configuration is optimized with respect to the width of the passband. The total electron dose is kept the same, but now evenly divided over 16 images, yielding an SNR per image which is 8 times lower than when two images are used. The defocus step between the first 8 images is $0.1 \mathrm{Sch}$ and between the last 8 images is $0.3 \mathrm{Sch}$. The start defocus is 0 Sch.

Comparing Fig. 8 with Fig. 7 shows the obvious merits of a specific choice of the free parameters because the same amount of electrons yields a much better overall precision. Likewise, results have been obtained for the spectrum of the real part of the exit wave. It should be noted that the estimated exit wave is computed by inversely Fourier transforming the estimated spectrum. This still requires regularization for the low and high spatial frequencies. 


\section{CONCLUSION}

This research illustrates the importance of experimental design for practical physical experiments. Careful choice of the free parameters may improve the precision of the quantities to be measured substantially. For the problem of exit wave reconstruction from TEM images by means of a defocus series, an expression for the precision of the reconstructed spectrum is derived. The free experimental parameters have been identified, and guidelines have been obtained as to how to choose their optimum values.

\section{REFERENCES}

[1] W. J. De Ruyter, "Quantitative high resolution electron microscopy and holography," Ph.D. dissertation, Delft Univ., 1992.

[2] M. A. Gribelyuk and J. L. Hutchinson, "On the iterative restoration of exit plane wave function from defocus series in HREM," in Proc. $49 \mathrm{th}$ Annu. Meeting Electron Microscopy Soc. of Amer., 1991, pp. 550-551.

[3] V. V. Fedorov, Theory of Optimal Experiments. New York: Academic, 1972.

[4] E. J. Kirkland, "Improved high resolution image processing of bright field electron micrographs I. Theory," Ultramicroscopy, vol. 15, pp. 151-172, 1984.

[5] E. J. Kirkland, B. M. Siegel, N. Uyeda, and Y. Fujiyoshi, "Improved high resolution image processing of bright field electron micrographs II. Experiment," Ultramicroscopy, vol. 17, pp. 87-104, 1986.

[6] A. J. Koster, A. van den Bos, and K. D. van der Mast, "An autofocus method for a TEM," Ultramicroscopy, vol. 21, pp. 209-222, 1987.

[7] M. A. O. Miedema, "Statistical design of exit wave reconstruction from a defocus series," Master's thesis, Delft Technical Univ., Dept. Physics, 1992.

[8] L. Reimer, Transmission Electron Microscopy. Springer series in Optical Sciences. Vol. 36. MacAdam, Ed. D. L. Berlin: Springer Verlag, 1984.

[9] W. O. Saxton, "Focal series restoration in HREM," in Proc. 1lth Int. Congress Electron Microscopy, Kyoto, Japan, 1986.

[10] W. M. Stobbs and W. O. Saxton, "Quantative high resolution transmission microscopy: The need for energy filtering and the advantages of energy-loss imaging," J. Microscopy. vol. 151, Pt. 2, pp. 171-184, Aug. 1988.

[11] A. van den Bos, "Parameter estimation," in Handbook of Measurement Science. New York: Wiley, 1982, ch. 8 .

[12] A. van den Bos, "Estimation of Fourier coefficients," IEEE Trans. Instr. Meas., vol. IM-38, no. 5, pp. 1005-1007, 1989.

[13] D. van Dyck and M. op de Beeck, in Proc. 12th Int. Congr. Electron Microscopy Seattle, I 26, 1991.
[14] R. H. Wade and J. Frank, "Electron microscope transfer functions for partially coherent axisl illumination and chromatic defocus spread," Optik, vol. 49, no. 1, pp. 81-92, 1977.

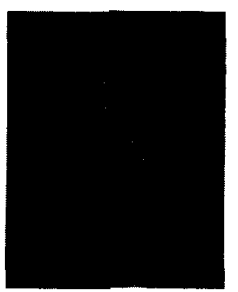

Martijn A. O. Miedema was born in 1966. He recieved the M.S. degree in applied physics from Delft University of Technology, The Netherlands, in 1992.

For his military service he joined the Royal Dutch Navy and is currently detailed with the TNO Physics and Electronics Laboratory, Scheveningen. His main research interest is in applying image analysis and image processing to measurement problems.

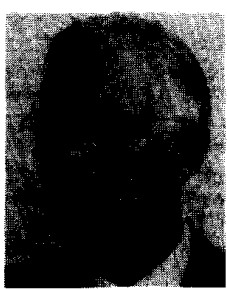

Adriaan van den Bos received the M.S. degree in applied physics and the D.Tech.Sc. degree from Delft University of Technology, Delft, The Netherlands, in 1962 and 1974, respectively.

From 1962 to 1964 he worked on instrumentation problems with the Institute of Perception of the Royal Defense Research Council, Soesterberg, The Netherlands. In 1964 he joined the Department of Applied Physics of Delft University of Technology where he is currently Full Professor. His main research interest is in applying parameter estimation to measurement problems in various fields of physics.

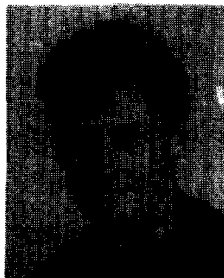

A. H. Buist was born in 1965. He received his Masters degree in Applied Physics (ir.) from Delft University of Technology, The Netherlands, in 1989.

Currently, he is in the process of writing his Ph.D. thesis in the Particle Optics Research Group of Delft University of Technology. His main research topic is the development of instrumentation and techniques for quantitative sample analysis in transmission electron microscopes. 